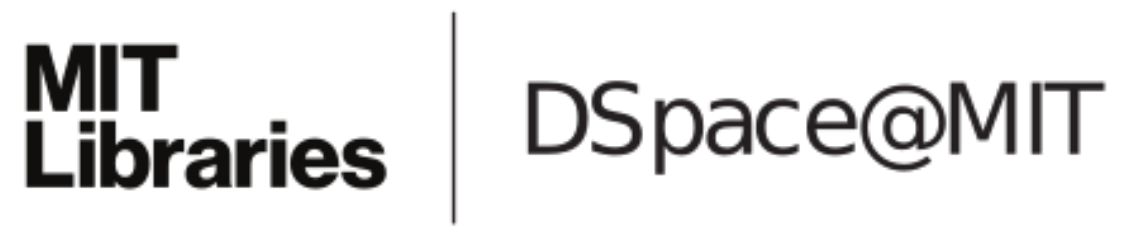

\author{
MIT Open Access Articles
}

Compliance and data quality in GPS-based studies

The MIT Faculty has made this article openly available. Please share how this access benefits you. Your story matters.

Citation: Isaacson, Michal, Noam Shoval, Hans-Werner Wahl, Frank Oswald, and Gail Auslander. "Compliance and Data Quality in GPS-Based Studies." Transportation 43, no. 1 lOctober 16, 2014): 25-36.

As Published: http://dx.doi.org/10.1007/s11116-014-9560-3

Publisher: Springer US

Persistent URL: http://hdl.handle.net/1721.1/103605

Version: Author's final manuscript: final author's manuscript post peer review, without publisher's formatting or copy editing

Terms of Use: Article is made available in accordance with the publisher's policy and may be subject to US copyright law. Please refer to the publisher's site for terms of use. 


\title{
Compliance and Data Quality in GPS-Based Studies
}

\author{
Michal Isaacson ${ }^{1}$,Noam Shoval ${ }^{2}$, Hans-Werner Wahl ${ }^{3}$, Frank Oswald ${ }^{4}$ and Gail \\ Auslander ${ }^{5}$
}

\footnotetext{
${ }^{1}$ New England University Transportation Center, AgeLab, Massachusetts Institute of Technology, 77 Massachusetts Avenue, E40-279, Cambridge, MA 02139

${ }^{2}$ Department of Geography, Hebrew University of Jerusalem, Mt. Scopus, Jerusalem 91905, Israel.

${ }^{3}$ Department of Psychological Ageing Research, Institute of Psychology, University of Heidelberg, Bergheimer Str. 20, 69115 Heidelberg, Germany.

${ }^{4}$ Interdisciplinary Ageing Research Group, Faculty of Educational Sciences, Goethe University, Frankfurt am Main, Grüneburgplatz 1, 60323 Frankfurt am Main, Germany

5 Paul Baerwald School of Social Work and Social Welfare, The Hebrew University of Jerusalem, Mt. Scopus, Jerusalem 91905 Israel.
} 


\begin{abstract}
Recent years have witnessed a growing volume of papers describing the use of GPS technology and other tracking technologies for obtaining data on time-space activities. These methods have several advantages over traditional methods of time-space data collection in terms of accuracy, resolution and length of the possible data collection period. However, to date, no work has been done on the compliance rates among participants and the resulting validity of the collected data. This paper presents a method that combines the use of a GPS receiver with Radio Frequency Identification (RFID) technology that was implemented in research on time-space activities of elderly persons with cognitive impairment.

The method presented in this paper enables monitoring the level of compliance of the participants during their participation in the study and presents a unique opportunity to examine the extent to which participants in a GPS based study are able to comply with study requirements. Healthy older adults and those with cognitive decline were found to be generally compliant with a complex study protocol. These results serve as another step in to the acceptance of GPS based studies as a valid methodology for mobility data collection.
\end{abstract}

Key words: Compliance, time-space activities, Global Positioning System, Data validity. 


\section{Introduction}

For many years the most common method for gathering information on human spatial activity has been the time-space diary. This method provides a systematic record of the way in which individuals occupy their time in space over a limited period, be it a few hours, a day, or a week (Anderson 1971). While time-space diaries have been used to great effect (see for example: Hanson and Hanson 1981; Goodchild and Janelle 1984; Janelle et al. 1988; Axhausen et al. 2002), they do have several disadvantages as research tools. In particular, time-space diaries require the subjects to be actively involved in the process of data collection by recording, in detail and at length, their activities throughout the entire experiment (Thornton et al. 1997).

In recent years, the rapid development and availability of small, inexpensive and reliable tracking devices, and most recently smartphones, has led to a growing volume of spatial research using such technologies (see for example: Macket et al. 2007; Shoval 2008; Wiehe et al. 2008, Shoval et al. 2013). Global Positioning Systems (GPS) devices offer researchers the opportunity for continuous and intensive high-resolution data collection in time (seconds) and space (meters) for extended periods of time. Aside from Transportation Studies (Wolf 2000; Wolf 2006; Oliveira et al. 2011), GPS and other tracking technologies are now used to collect time-space data in a wide variety of fields including tourism (Shoval and Isaacson 2007; Xia 2008), environmental health (Phillips et al. 2001; Elgethun et al. 2003), and in medicine areas such as Alzheimer's disease (Miskelly 2004; 2005), physiology (Terrier and Schutz 2005) and cardiology (Le Faucheur et al. 2008).

GPS assisted data collection is especially useful for collecting time-space data over long periods of time. The ease of use and the low burden on the participants make this a viable tool for data collection spanning over several days or weeks. As this data collection method becomes more and more popular the research community must 
concern itself with the validity of the data being collected. We must ask ourselves, to what degree are respondents able to fulfill the requirements of participating in a GPS survey and remember to take the GPS unit with them when they leave their house?

One of the main drawbacks associated with GPS assisted data collection is in the active role that participants need to take in insuring high data quality. Participants have to remember to take the GPS unit with them when they leave their homes, and in the case of studies that last for several weeks, the participants must remember to charge the device. Unlike travel diaries, if a participant forgets to take his GPS with him when leaving home, there is little or no opportunity to fill in the data that is missing due to this mishap.

When using travel diaries as a data collection tool, Axhausen et al. (2007) have shown that there is little respondent fatigue. They demonstrated this using data from travel behavior studies that use travel diaries and span over a period of several weeks. Their study demonstrated that there is actually a learning tendency that is found as an increase in the report of trips as the study progresses. Previous studies have explored the discrepancies between GPS tracked trips and survey reported trips and found patterns of discrepancy associated with age, trip purpose and number of trips (Stopher and Shen 2011; Bricka et al. 2012). Younger participants and 'heavy travelers' reported fewer trips than the GPS recorded. Work trips tend to be over reported while people were found to underreport non-work trips.

When exploring missing data it is extremely important to understand the nature and the distribution of missing data in GPS assisted surveys in order to avoid information bias. It is easy for researchers to get a false sense of confidence in the quality of the data that they had collected. For example, if the GPS was located in the participant's home from three p.m. every afternoon until the next morning, this could 
mean that the participant does not leave his or her home in the evening. Alternatively, it could also mean that the participant spends his or her nights partying in clubs but prefers not to take the unit with him or her. In this example, the data on the whereabouts of the participant are not only missing, they are actually incorrect, resulting in systematic error in the collected data. In sum, compared to more traditional time-space diaries, GPS devices have the potential for more complete data collection, but their use is also subject to similar potential biases.

This paper is a by-product of "SenTra" a bi-national Israeli-German research project that examined the relationship between cognitive decline and mobility. The study enlisted older adults with different levels of cognitive impairment, ranging from a control group of cognitively healthy to people diagnosed with mild dementia. After completing a battery of cognitive tests in a clinical setting, participants were interviewed in their homes and asked to carry a GPS receiver with them when they leave their home for a duration of four weeks (for the full study protocol see Shoval et al. 2008).

Naturally, data validity was a major concern for a project of this character. As researchers, we asked ourselves: How can we design a study based on a high level of compliance from people who have just had cognitive decline clinically demonstrated. How can we ask this population to remember to carry a GPS unit with them? Being aware of the cognitive challenges that these people face, how can we treat data collected in this manner as valid data that describes mobility? How can we trust the self-reporting of participants that claim to be actively participating in the study but who might not be able to actually remember whether they fulfilled the study requirements?

A solution to these problems was found in an innovative technology developed in the context of law enforcement and later adapted to a health care context. The technological solution, described at length later in this paper, used Radio Frequency 
Identification (RFID) technology to indicate whether the participant left home without the GPS unit. As researchers, we were not able to eliminate missing data, but we were able to achieve a second best, to know when data was missing and be able to assess the validity of the data collected. This novel study design enabled a unique opportunity and allowed a window into examining GPS assisted survey's data validity.

This paper reports on the validity of the GPS data collected and tries to answer the question: To what degree are older adults able to fulfill the requirements of a GPS assisted study?

The remainder of this paper is divided into three sections. First, the data collection method and the unique combination of technologies that was used is presented. Then the results of the study are introduced and discussed. The conclusion of the paper discusses the significance that these findings have for planning and executing GPS based studies.

\section{Method}

\section{Data collection}

The GPS tracking kit (purchased from HomeFree Wireless TelehomeCare Solutions, Tel-Aviv, Israel) consists of three main elements: 1) the STAR unit, which needs to be taken when the participant leaves home. The unit weighs 400 grams and contains a GPS receiver, GSM modem and an RF receiver; 2) a waterproof wristwatch that includes a Radio Frequency (RF) transmitter and additional sensors including one that detects whether the watch is being worn on a person's body; 3) a stationary home unit that repeats the RF signal and allows the participant to walk freely around the house (Figure 1). The waterproof "wristwatch" RF transmitter allows the researchers to know whether participants are complying with the research participation guidelines and 
whether they are carrying the GPS device at any given moment. If the strap is opened or the RF transmitter is not in contact with the body, the system issues a notification. If the watch is worn on the hand, but is farther than ten meters from the GPS receiver (at home this maximum distance is 70 meters because of the home monitoring unit) then a notification is also issued. These notifications allow the researchers to determine whether the research subject is participating at a satisfactory level. Both notifications of a missing body signal for the wristwatch or a distance of more than 10/70 meters (outside/at home) indicate that there is a problem with the participant's compliance. If the wristwatch is removed we lose certainty as to the whereabouts of the participant. Similarly, if the STAR unit is more than 10 meters from the wristwatch it means that the person has moved away from the GPS unit and the locations obtained by the GPS no longer represent the person's location. Aggregating these data allows for measuring the overall level of compliance and, as a result, to assess the quality of the data obtained during the relatively long tracking period.

\section{Figure 1: Elements of the location kit}
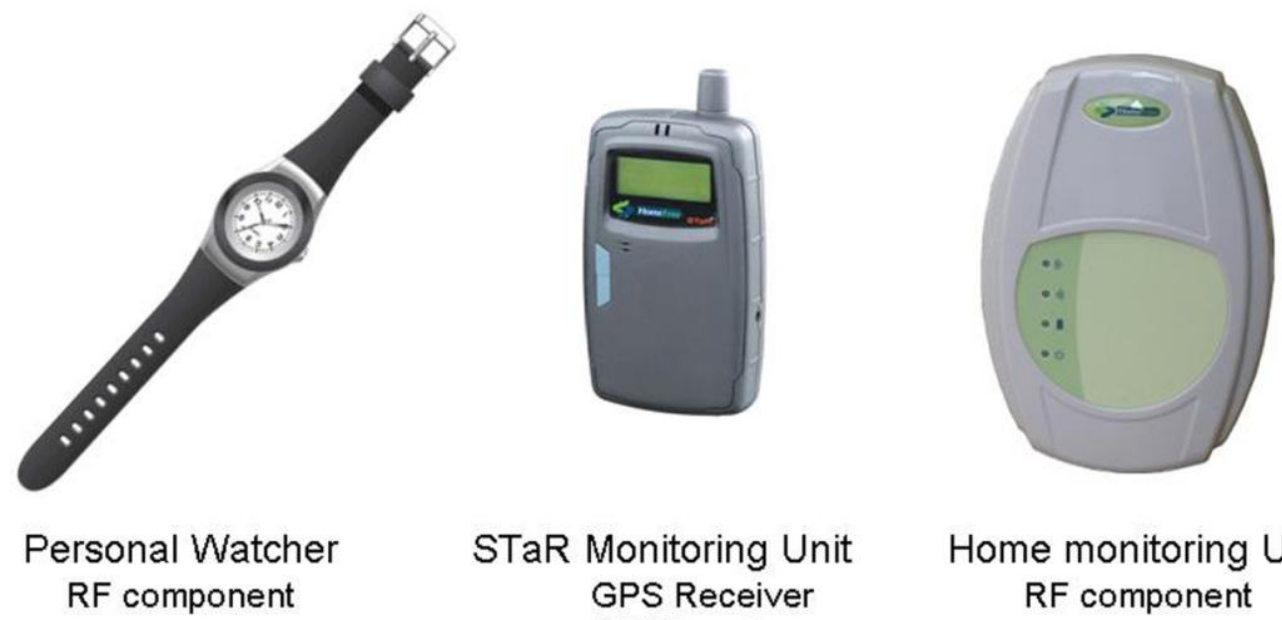

STaR Monitoring Unit

GPS Receiver GSM modem Home monitoring Unit RF component RF component 
The STAR unit was programmed to obtain locations every 10 seconds when the participant was outside the home. It had an ability to function for about 12 hours without recharging. This is an important feature since some participants leave their homes for long periods of time during the day. The data collected were transferred by GPRS (General Packet Radio Service) to the project's server; this enabled us to monitor the tracking kits in real-time in order to ascertain that the kit was functioning, that it had sufficient battery power, and that the subject was participating in the research as expected.

Sample

Data analyzed in this paper were drawn from the Israeli part of the SenTra study, which was completed at the beginning of 2011. Eighty nine older adults participanted in the study and were tracked for 28 days. The participants were selected from two groups, older adults with mild cognitive decline or mild dementia and a control group of older adults who were tested and proven to be cognitively healthy. Participants with cognitive decline were recruited through the Memory Clinic at the Tel-Aviv Sourasky Medical Center, while healthy controls were recruited by combining advertisements in the media and a snowball sampling technique. Additional exclusion criteria included mobility impairments as well as any underlying mental or physical diseases (for full details of the study protocol see Shoval et al. 2008). All participants were given a full explanation of the study, its purpose, and their role. Participants could withdraw from the study at any point in time.

The sample described in table 1, consisted of 89 men and women, 63-92 years old (53 cognitively impaired, 39 healthy). Participants with cognitive impairment were somewhat older than healthy controls, although the difference was not statistically 
significant. There was no difference by gender; however, healthy controls were more educated than the cognitively impaired participants. For a more detailed presentation of the study design and the clinical classification criteria please see Shoval et al. 2008.

A few words about Mild Cognitive Impairment (MCI) are in order. MCI is a condition in which a degree of cognitive decline that is greater than expected for a person's age and education is demonstrated. This degree of cognitive decline does not interfere with day-to-day life (Gauthier et al. 2006). In lay terms the physician that screen the participants in this study described people with MCI as people that are late for their appointments because they had trouble finding parking. These people are still active many of them still drive and maintain a full schedule of activities; these people are considered by themselves and by those that surround them as healthy and not as people with a disease. From an epidemiological point of view, the prevalence of the condition ranges from 3\% to $19 \%$ among adults over the age of 65 .

Table 1: The two groups of participants by gender and age $(n=89)$

\begin{tabular}{|l|l|l|}
\hline & $\begin{array}{l}\text { Healthy } \\
\text { N }(\%)\end{array}$ & $\begin{array}{l}\text { Cognitive Decline } \\
\text { N }(\%)\end{array}$ \\
\hline Gender & & \\
\hline Male $(\mathrm{N}=42)$ & $\mathbf{1 4}(40)$ & $\mathbf{2 8}(51.9)$ \\
\hline Female (N=47) & $\mathbf{2 1}(60)$ & $\mathbf{2 6}(48.1)$ \\
\hline Age Mean Age (sd) 77.3 (6.7) & $\mathbf{1 9}(54.3)$ & $\mathbf{1 9}(35.2)$ \\
\hline 62-76 years (N=38) & $\mathbf{1 6}(45.7)$ & $\mathbf{3 5}(64.8)$ \\
\hline 77-92 years (N=51) & $\mathbf{3 5}(100.0)$ & $\mathbf{5 4}(100.0)$ \\
\hline Total &
\end{tabular}


The collected data was assessed using a two-step method. First, each hour of the day was classified as valid or invalid. In order for an hour to be valid, it had to have at least 30 minutes of compliance with the study's requirements; the participant had to be wearing the watch and to be located in the same place as the STAR unit. The threshold was aimed at discerning between technical malfunction and noncompliant behavior.

Next, each day was classified as valid or invalid. A valid day of participation had to have a full 24 hours of compliance with the study requirements. This strict requirement takes into account the disparity between the ease of complying with the study requirements while at home and the difficulty of complying when leaving home. While at home, it was easy for participants to be compliant, they only had to wear the wristwatch. When leaving home, one had to remember to take the STAR unit with them, making it more difficult to comply with the study requirements. A missing hour of compliance when a person is out of their home indicates missing mobility data, making it impossible to calculate daily volumes of spatial activity. That said, days that have hours of missing data still contain valuable information regarding the spatial activity that was observed. The data can be used in assessing activities and mobility patterns that were observed while remaining cautious, especially when examining volumes of mobility behavior, keeping in mind that there may be missing data.

\section{Results}

The levels of compliance are presented below divided into the subsamples based on cognitive function. The subsamples with demonstrated cognitive decline do not exhibit the behavior of a cognitively healthy population but they rather present the extreme case in which we might expect low levels of compliance, due to memory decline. One can expect this to represent the worst case in which participants have a recognized condition that interferes with their ability to remember to carry the GPS unit with them. 
The average number of valid days (table 2) is highest for the healthy control group, is slightly lower for the participants with cognitive decline. This decline in valid data reveals the expected difficulty that people with Cognitive Decline have in complying with the study's requirement of remembering to take the GPS unit with them when they leave their home. While this difficulty is expected despite what we might have anticipated the participants were able to participate to some degree and a substantial amount of data describing their spatial activity was collected.

Tables 2 and 3 demonstrate that there is very little association between gender and compliance. The compliance rates among the older cohort were slightly higher than the younger group, indicating that there is no decline in compliance with age. A t test did not reveal a significant difference in the ability to participate based on age or gender. 
Table 2: Average Percent of Valid Days (gender)

\begin{tabular}{cccc}
\hline Gender & $\begin{array}{c}\text { Healthy } \\
\text { avg (stdv) }\end{array}$ & $\begin{array}{c}\text { Cognitive } \\
\text { Decline }\end{array}$ & Total \\
\hline Male & $41.91(28.13)$ & $36.87(25.07)$ & $38.67(25.98)$ \\
Female & $43.88(27.32)$ & $37.33(28.24)$ & $40.48(27.71)$ \\
Whole Sample & $\mathbf{4 3 . 1 2 ( 2 7 . 2 8 )}$ & $\mathbf{3 7 . 1 0 ( 2 6 . 4 1 )}$ & $\mathbf{3 9 . 6 5 ( 2 6 . 8 )}$ \\
\hline
\end{tabular}

Table 3: Average Percent of Valid Days (age)

\begin{tabular}{cccc}
\hline Age & Healthy & $\begin{array}{c}\text { Cognitive } \\
\text { Decline }\end{array}$ & Total \\
\hline Younger Old (63-76) & $38.72(29.06)$ & $36.00(27.18)$ & $37.36(27.79)$ \\
Older Old (77-92) & $47.31(25.5)$ & $37.72(26.37)$ & $41.27(26.23)$ \\
Whole Sample & $\mathbf{4 3 . 1 2 ( 2 7 . 2 8 )}$ & $\mathbf{3 7 . 1 0 ( 2 6 . 4 1 )}$ & $\mathbf{3 9 . 6 5 ( 2 6 . 8 )}$ \\
\hline
\end{tabular}

When considering the hours of compliance with the study requirements the rates are a lot higher. This is because an hour can be categorized as valid even if the hours before or after are not while an invalid hour rules the whole day as invalid. Examining hours of compliance gives an indication as to the robustness of the spatial data collected. On average, people of all groups were proven compliant around $80 \%$ of the time. As in the case of valid days of participation, there is no significant difference between the two groups in relation to days of compliance. Age and gender (tables 4 and 5) are also not found to be associated with compliance rates when measured by hour.

Table 4: Average Percent of Valid Hours (gender) 


\begin{tabular}{cccc}
\hline Gender & Healthy & $\begin{array}{c}\text { Cognitive } \\
\text { Decline }\end{array}$ & Total \\
\hline Male & $81.73(14.12)$ & $80.30(17.99)$ & $80.81(16.57)$ \\
Female & $80.09(20.34)$ & $78.60(20.41)$ & $79.31(20.19)$ \\
Whole Sample & $\mathbf{8 0 . 7 2 ( 1 8 . 0 4 )}$ & $\mathbf{7 9 . 4 6 ( 1 9 . 5 )}$ & $\mathbf{8 0 . 0 0 ( 1 8 . 5 4 )}$ \\
\hline
\end{tabular}

Table 5: Average Percent of Valid Hours (age)

\begin{tabular}{cccc}
\hline Age & Healthy & $\begin{array}{c}\text { Cognitive } \\
\text { Decline }\end{array}$ & Total \\
\hline Younger Old (63-76) & $76.08(23.03)$ & $81.09(17.28)$ & $78.59(20.24)$ \\
Older Old (77-92) & $85.14(10.3)$ & $78.55(20.17)$ & $80.99(17.37)$ \\
Whole Sample & $\mathbf{8 0 . 7 2 ( 1 8 . 0 4 )}$ & $\mathbf{7 9 . 4 6 ( 1 9 . 5 )}$ & $\mathbf{8 0 . 0 0 ( 1 8 . 5 4 )}$ \\
\hline
\end{tabular}

Figure 2 presents the rate of compliance over different days of the week by examining compliant hours and days. There was a statistically significant difference between the hours of compliance on different days of the week as determined by oneway ANOVA $(F(6,2521)=2.767, p=.011)$. A Tukey post-hoc test revealed that there is a statistically significantly lower rate of compliance on Saturday compared to Thursday. There are no statistically significant differences between the intermediate and advanced groups. A similar test revealed that there is no statistically significant differences between the days of the week when whole days are classified as compliant or non-compliant.

Figure 2: Percentage of compliant days and compliant hours by day of the week $(\mathbf{n}=\mathbf{8 9})$ 


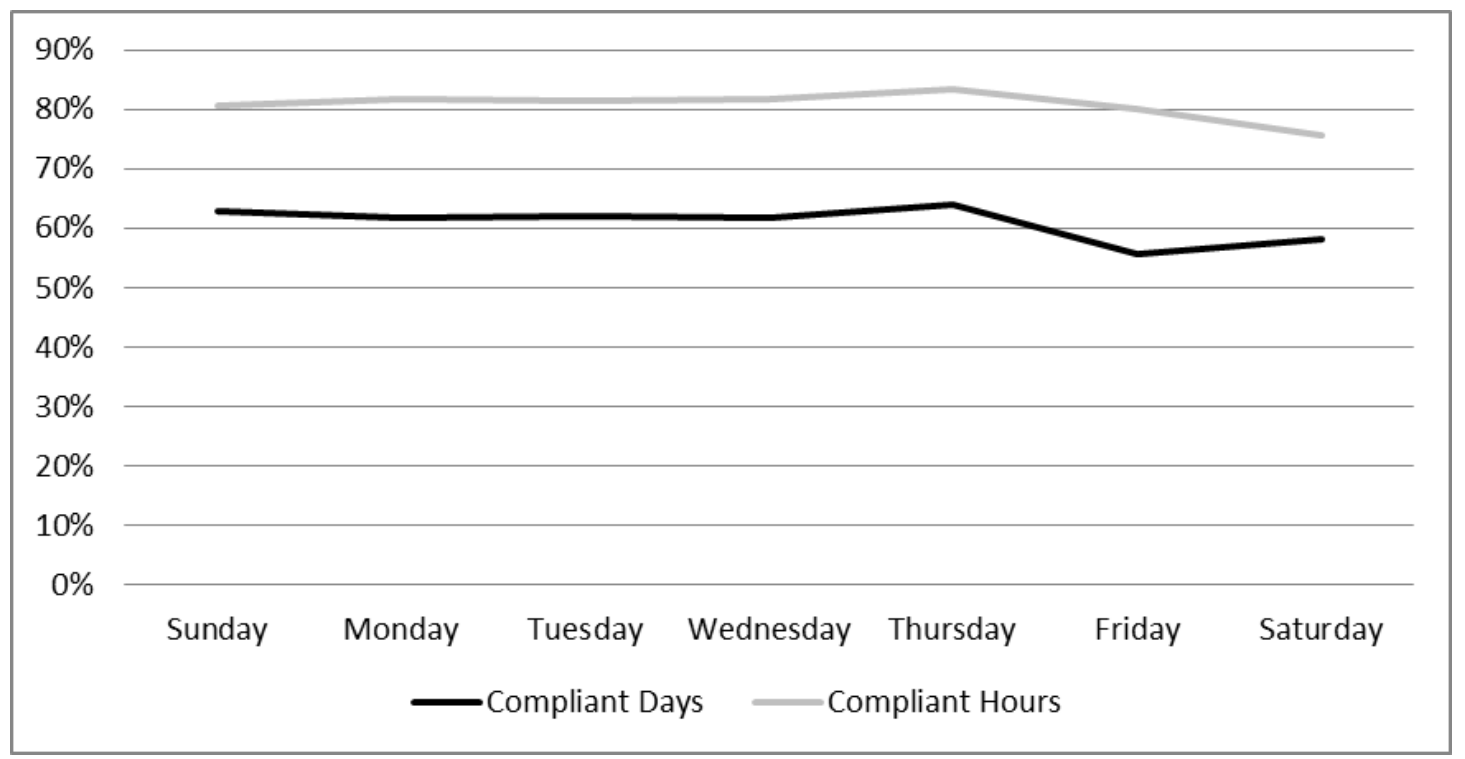

Examining rates of compliant hours and valid days over the 28 days of participation (figure 3) eliminates the possibility of there being a fatigue effect in the collected data. Participants neither present a trend of learning (as reported by Axhausen et al. 2007) which would be indicated in rising rates of compliance as the study progresses nor does there seem to be demonstrated sampling fatigue over the period of participation. 


\section{Figure 3: Percentage of compliant days and compliant hours by day of participation $(\mathbf{n}=89)$}

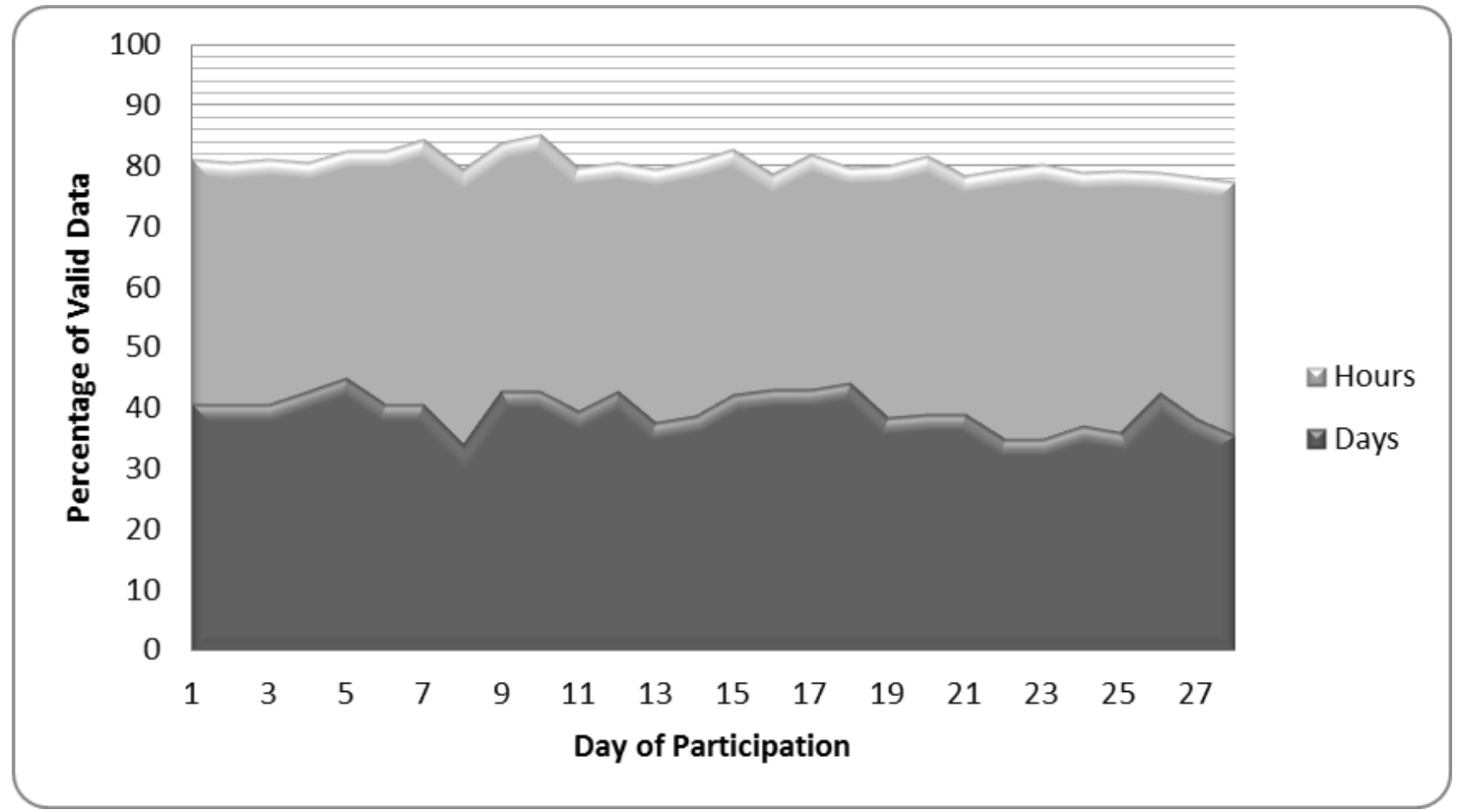

\section{Discussion}

Combining the technologies that this study used, gives us the opportunity to further analyze the source of the problem that led to the lack of valid data. There are four sources of possible invalid data:

1. The participant left home without the GPS unit. This would be indicated by the system when the watch loses communication with the STAR unit.

2. The participant removed the wrist watch from their arm. In this situation the participant might still comply with the study requirement of taking the STAR unit with them when leaving home but since we would have no way of knowing if they left home or not for the purposes of this study it would be considered invalid data.

3. The STAR unit ran out of power. This situation can be caused by a lack of compliance, in the case when the participant did not charge the STAR unit at 
night as required. This failure could also be caused by technical failure, for example, a battery with a weak charge or a software malfunction.

4. Technical problems such as communications problems can cause a loss of data resulting in a time of uncertainty regarding the participant's compliance.

The first two reasons for invalid data indicate a lack of compliance with the study requirements. The fourth reason for invalid data is not associated with the participants' behavior and therefore does not indicate a lack of compliance with the study requirements. The third scenario can be caused by the participant or by the equipment. Table 6 shows the percentage of hours that were invalid and the reasons for the invalidity. There is no significant difference between the people with cognitive decline and the control group when examining the different reasons for data invalidity. Overall, $81 \%-88 \%$ of the time participants were compliant with the study requirements.

Table 6: Rates of invalid data, as percentage of participation time $(\mathrm{n}=89)$

\begin{tabular}{ccccc}
\hline & Took off watch & Forgot GPS & Battery failure & $\begin{array}{c}\text { Total percent } \\
\text { of invalid } \\
\text { data }\end{array}$ \\
\hline Cognitive Decline & $7.79 \%$ & $4.67 \%$ & $6.76 \%$ & $19.22 \%$ \\
\hline Healthy & $6.82 \%$ & $4.59 \%$ & $6.93 \%$ & $18.34 \%$ \\
\hline Whole Sample & $7.42 \%$ & $4.64 \%$ & $6.82 \%$ & $18.88 \%$ \\
\hline
\end{tabular}

One might postulate that there is an association between the time of day and compliance with the study requirements. Being compliant during the day, when participants tend to be active, requires that the participant actively remember to take the GPS unit with them while at night compliance can be obtained passively while sleeping in bed. Figure 4 plots the different statuses of compliance that a participant can have 
throughout a 24 hour cycle while differentiating between the participants with cognitive decline and those who are cognitively healthy.

Examining the figure reveals that there are only small differences in levels of participation between participants with cognitive decline and those without cognitive decline. The data reveals that participants found it the hardest to keep the wrist watch on. This strain was especially prominent in the late evening and earlier morning a time when most participants were sleeping. These times have the highest percentage of noncompliant data due to participants removing the watch. This was the main complaint that participants would raise when asked by the research staff about their experience participating in this study. The positive side of this is that while taking of the watch at night does not comply with the research protocol it also does not affect the quality of the spatial data collected.

While not statistically significant the data does indicate higher levels of forgetting the GPS unit at home, during hours of high activity, among those with cognitive decline. This can be seen as a manifestation of the disease with which this population is dealing with and can be understood as part of the implications of working with people with cognitive decline. 
Figure 4: Percentage of valid and invalid data throughout a 24 hour cycle for cognitively healthy and cognitively impaired participants.

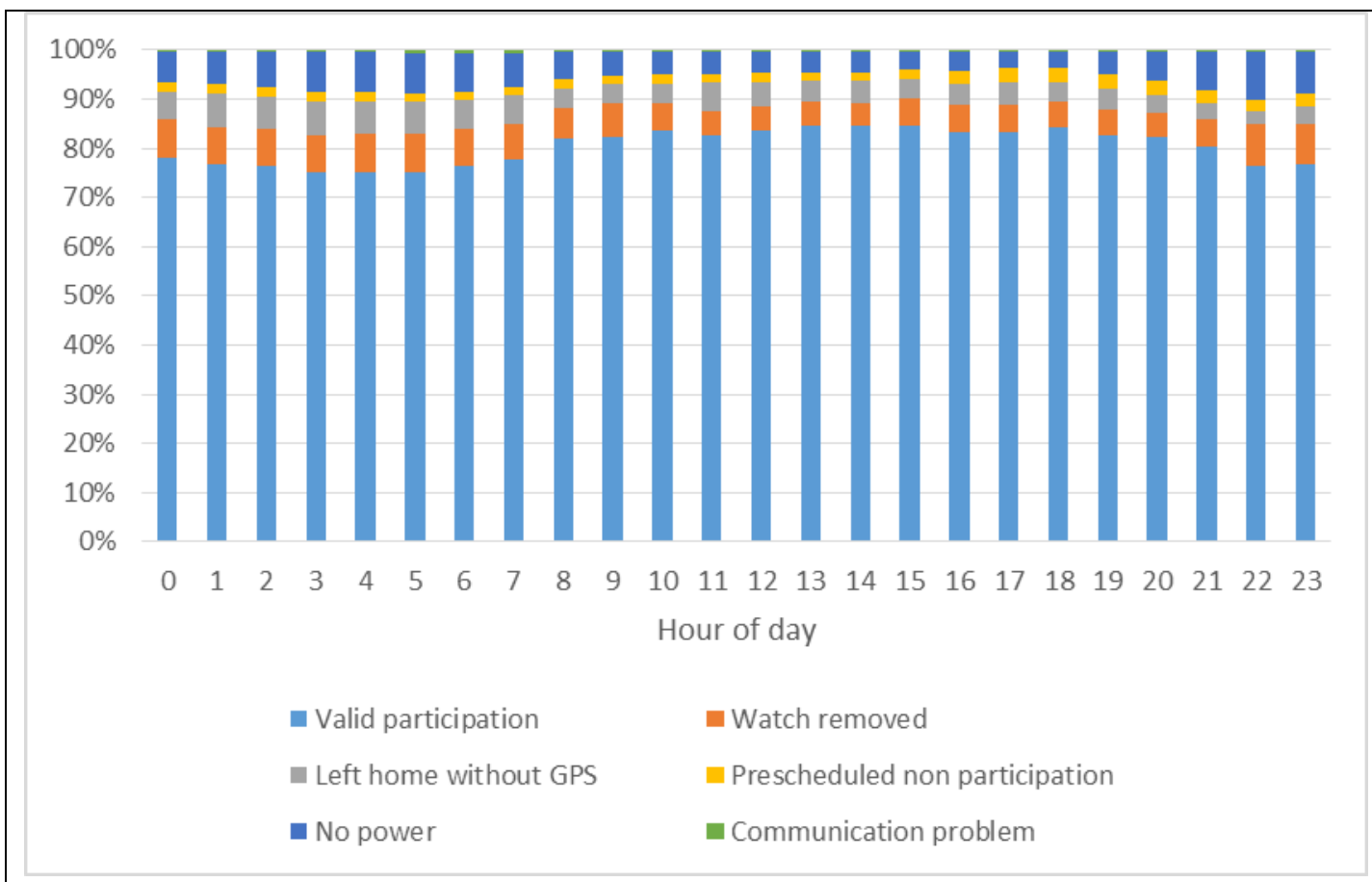

4a-Cognitively Healthy participants

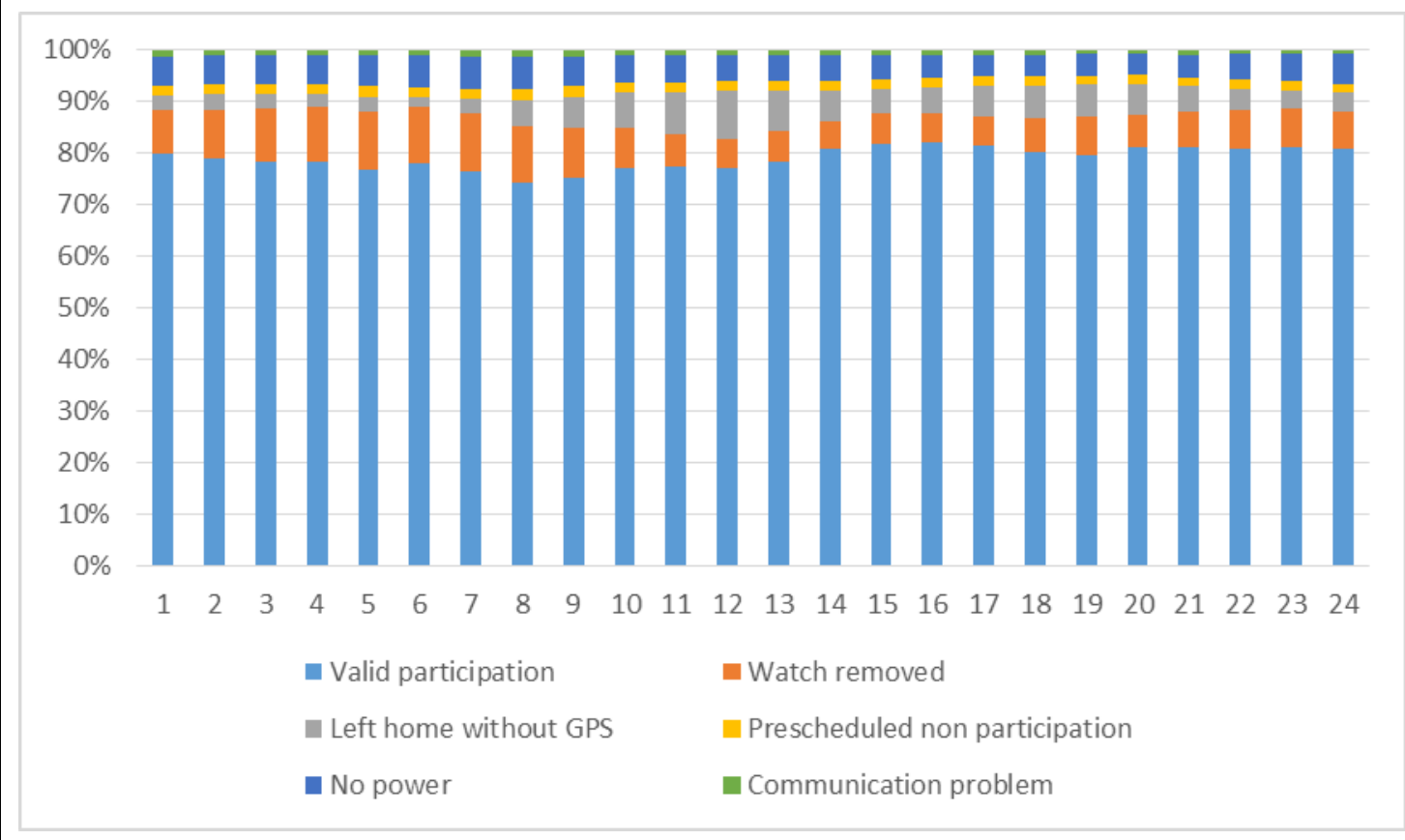

$4 b$ - Participants with cognitive decline 


\section{Conclusion}

Previous studies that have tried to quantify and rate the quality of time-space data collected mostly using time-space diaries have examined days of immobility as an indicator of the amount of soft refusals accrued from the participants in the study (Madre et al. 2007). Most data collection methodologies, both GPS based and time-space diaries, make it is impossible to discern between days of immobility and soft refusals. This has led travel diary surveys to exclude certain populations (including older adults) as a matter of policy.

This paper demonstrated a method with which it is possible to measure compliance rates in GPS based studies. The results reported in this paper took advantage of a unique opportunity to glimpse into compliance rates among participants in GPS based studies. This opportunity was made possible by the combination of several technologies in a way that is not usually used in research design. In most cases, using these technologies is not a feasible study design due to considerations of cost and ease of use.

To what extent do these results represent trends in a more heterogeneous sample? The population sampled in this study is unique, older adults some of whom suffer from cognitive decline. People with cognitive decline are generally a population that many studies steer away from because of the methodological challenges associated with data collection. How can we expect the compliance of this population to compare to a more heterogeneous sample? Failure to comply with study requirements in a GPS assisted data collection effort are a result of both soft refusals as well as occasional forgetfulness. One can argue that this sample represents an extreme case in which forgetfulness is more prevalent than it would be among those without cognitive decline. We see no reason to believe that soft refusal rates are any different than they would be in 
a more heterogeneous sample. In addition the compliance rates reported in this paper can be used as bench marks when estimating rates of compliance in future studies.

The findings of this study reveal the feasibility of the rather complex data assessment approach used. Though there were differences between the compliance levels among different sub-groups, many of the participants were able to comply with the study requirements. The result of this data collection procedure is the creation of a database with high-resolution data about time-space activities of elderly people over a period of one month. In addition we have demonstrated that participants with cognitive decline who are generally considered to be a difficult population to collect data from because of memory problems and difficulty complying with research protocols, can actually fulfill the requirements of participating in a GPS based data collection effort. This lends to the assumption that GPS based data collection efforts in a more heterogeneous population have high compliance rates.

\section{REFERENCES}

Anderson, J (1971) Space-time budgets and activity studies in urban geography and planning. Environment and Planning A 3(4), 353-368.

Axhausen, K.W. and Madre, J-L. (2002) Share of Immobiles in Travel Diary Surveys: A Review. ETH Report 133, Swiss Federal Institute of Technology, Zurich.

Axhausen, K., Zimmermann, A., Schönfelder, S., Rindsfüser, G., and Haupt, T. (2002) Observing the rhythms of daily life: A six-week travel diary. Transportation. 29: 95-124.

Axhausen, K.W., M. Löchl, R. Schlich, T. Buhl and P. Widmer (2007) Fatigue in long duration surveys, Transportation, 34 (2) 143-160.

Bricka, S. G., Sen, S., Paleti, R., and Bhat, C. R. (2012). An analysis of the factors influencing differences in survey-reported and GPS-recorded trips. Transportation Research Part C: Emerging Technologies, 21(1), 67-88.

Elgethun, K, Fenske, R A, Yost, M G and Palcisko, G J (2003) Time-location analysis for exposure assessment studies of children using a novel global positioning system instrument. Environmental Health Perspectives 111, 115-122. 
Gauthier S, Reisberg B, Zaudig M, Petersen RC, Ritchie K, Broich K, et al. (2006) Mild cognitive impairment. Lancet 367:1262-70.

Goodchild, M F and Janelle, D G (1984) The city around the clock: space-time patterns of urban ecological structure (Halifax, Canada). Environment and Planning A 16, 807-820.

Hanson, S. and P. Hanson. 1981. The activity patterns of urban residents: Dimensions and relationships to sociodemographic characteristics. Economic Geography 57:332-347.

Janelle, D G, Goodchild, M F and Klinkenberg, K (1988) Space-time diaries and travel characteristics for different levels of respondent aggregation. Environment and Planning $A$ 20(7), 891-906.

Le Faucheur, A., Abraham, P., Jaquinandi, V., Bouyé, P., Saumet, J. L., and NouryDesvaux, B. (2008) Measurement of Walking Distance and Speed in Patients With Peripheral Arterial Disease. Circulation: Journal of the American Heart Association, 117: 897904.

Macket, R., Brown, B., Gong, Y., Kitazawa, K., and Paskins, J. (2007) Children's Independent Movement in the Local Environment. Built Environment, 33 (4): 454-468.

Madre, J.-L., K.W. Axhausen and W. Brög (2007) Immobility in travel diary surveys, Transportation, 34 (1) 107-128.

Miskelly F (2004) A novel system of electronic tagging in patients with dementia and wandering. Age and ageing, 33(3):304-306.

Miskelly F (2005) Electronic tracking of patients with dementia and wandering using mobile phone technology. Age and ageing, 34(5):497-499.

Oliveira, M., P. Vovsha, J. Wolf, Y. Birotker, D. Givon, J. Paasche. (2011) GPS-Assisted Prompted Recall Household Travel Survey to Support Development of Advanced Travel Model in Jerusalem, Israel. Transportation Research Record 2246, pp. 16-23.

Phillips, M L, Hall, T A, Esmen, N A, Lynch, R and Johnson, D L (2001) Use of global positioning system technology to track subject's location during environmental exposure sampling. Journal of Exposure Analysis and Environmental Epidemiology, 11, 207-215.

Shoval, N and Isaacson, M (2006) The application of tracking technologies to the study of pedestrian spatial behaviour, The Professional Geographer 58(2), 172-183.

Shoval, N. (2008) Tracking Technologies and Urban Analysis. Cities, vol. 25, 1:21-28.

Shoval, N., Auslander, G. K., Freytag, T., Landau, R., Oswald, F., Seidl, U., Wahl, H.-W., Werner, S. and Heinik, J. (2008) 'The use of advanced tracking technologies for the analysis of mobility in Alzheimer's disease and related cognitive diseases', BMC Geriatrics, 8: 7.

Shoval, N., Kwan, M-P., Reinau, K. H., and Harder, H. (Accepted 9.9.2013) The 
Shoemaker's Sons Always Goes Barefoot: Implementations of GPS and other tracking technologies for geographic research, Geoforum.

Stopher, P., and Shen, L. (2011). In-Depth Comparison of Global Positioning System and Diary Records. Transportation Research Record: Journal of the Transportation Research Board, (2246), 32-37.

Terrier, P., and Schutz, Y. (2005) How useful is satellite positioning system (GPS) to track gait parameters? A review. Journal of NeuroEngineering and Rebabilitation. 2:28.

Thornton, P R, Williams, A M and Shaw, G (1997) Revisiting time-space diaries: an exploratory case study of tourist behaviour in Cornwall, England. Environment and Planning A, 29(10), 1847-1867.

Wiehe, S.E., Hoch, S. C., Liu, G. C., Carroll, A. E., Wilson, J. S., and Fortenberry, J. D. (2008) Adolescent Travel Patterns: Pilot Data Indicating Distance From Home Varies by Time of Day and Day of Week. Journal of Adolescent Health, 42(4): 418-420.

Wolf, J. (2000) Using GPS Data Loggers to Replace Travel Diaries in Collection of Travel Data. Dissertation, Georgia Institute of Technology, School of Civil and Environmental Engineering, Atlanta, Georgia.

Wolf, J. (2006) Applications of New Technologies in Travel Surveys in Travel Survey Methods: Quality and Future Directions. Edited by P. Stopher and S. Stecher, Chapter 29, pp. 531-544.

Xia, J., Arrowsmith, C., Jackson, M., and Cartwright, W. (2008) The wayfinding process relationship between decision-making and landmark utility. Tourism Management, 29: 445457.

Michal Isaacson is a postdoctoral associate at the MIT AgeLab. Trained as an Urban Geographer her work uses advanced tracking technologies to study human spatial behavior throughout the lifespan.

Noam Shoval is an Associate Professor at the Department of Geography, The Hebrew University of Jerusalem, Israel. His research focuses in recent years on the implementation of tracking technologies in various areas of spatial research such as tourism, urban studies and medicine.

Hans-Werner Wahl is Professor of Psychological Aging Research at the Institute of Psychology, Heidelberg University, Germany. His research activities include the understanding of the role of physical-technological environments for aging well, adaptational processes in the context of age-related chronic functional loss, processes of awareness of aging in a lifespan perspective, and intervention research.

Frank Oswald is Professor for Interdisciplinary Ageing Research at the Goethe University Frankfurt, Germany. He is a psychologist by training and co-author of 
several articles in the area of Environmental Gerontology, addressing issues of p-e transaction, contexts of adult development, housing and relocation.

Gail Auslander is Zena Harman Professor, Paul Baerwald School of Social Work and Social Welfare, Hebrew University of Jerusalem. Her research focuses on psychosocial aspects of health and illness, including the role of family caregiving to elderly people in the community and in-patient settings.

Acknowledgements

Support for this project provided by the German Federal Ministry of Education and Research (BMBF) within the framework of German-Israeli Project Cooperation (DIP) is gratefully acknowledged. Three reviewers provided valuable input on an earlier version of the paper. However, the authors are solely responsible for any errors and mistakes. 